SOI: 1.1 /TAS DOI: $10.15863 /$ TAS

International Scientific Journal Theoretical \& Applied Science

p-ISSN: 2308-4944 (print) $\quad$ e-ISSN: 2409-0085 (online)

Year: $2015 \quad$ Issue: 08 Volume: 28

Published: $30.08 .2015 \quad \underline{\text { http://T-Science.org }}$

SECTION 26. Radio-technique. Electronics. Telecommunications.
Marina Yur'evna Zvezdina Doctor of Physical and Mathematical Sciences, associate professor head of the department of Don State Technical University, Russia, Rostov-on-Don zvezdina_m@mail.ru

Yulia Aleksandrovna Shokova Candidate of Physical and Mathematical Sciences, associate professor of Don State Technical University, Russia, Rostov-on-Don

Andrey Viktorovich Shokov postgraduate student of Don State Technical University, Russia, Rostov-on-Don

Maksim Andreevich Andronov student of Don State Technical University, Russia, Rostov-on-Don

Vladimir Igorevich Tyul'pin student of Don State Technical University, Russia, Rostov-on-Don

\title{
VISUAL REPRESENTATION OF ENERGY FLUX DENSITY IN THE BACKSPACE OF CIRCULAR APERTURE REFLECTOR ANTENNA FOR MOBILE COMMUNICATIONS
}

\begin{abstract}
The results of electromagnetic situation research in the backspace of mobile system reflector antennae are given. It is shown that in spite of mirror construction details the energy flux value in antenna backspace at phase center level is twice as big as the permissible exposure limit. The visualization results for energy flux density distribution are given, the possible implementation is shown.

Key words: energy flux density, reflector antenna, visual representation of field structure, circular aperture, backspace, diffraction area.

Language: Russian

Citation: Zvezdina MY, Shokova YA, Shokov AV, Andronov MA, Tyul'pin VI (2015) VISUAL REPRESENTATION OF ENERGY FLUX DENSITY IN THE BACKSPACE OF CIRCULAR APERTURE REFLECTOR ANTENNA FOR MOBILE COMMUNICATIONS. ISJ Theoretical \& Applied Science 08 (28): 5664.

Soi: http://s-o-i.org/1.1/TAS-08-28-8 Doi: crossef http://dx.doi.org/10.15863/TAS.2015.08.28.8

\section{ВИЗУАЛИЗАЦИЯ РАСПРЕДЕЛЕНИЯ ПЛОТНОСТИ ПОТОКА ЭНЕРГИИ В ЗАДНЕМ ПОЛУПРОСТРАНСТВЕ ЗЕРКАЛЬНОЙ АНТЕННЫ С КРУГЛОЙ АПЕРТУРОЙ СИСТЕМЫ ПОДВИжНОЙ СВЯЗИ}

Аннотация: Приводятся результаты исследований электромагнитной обстановки в заднем полупространстве зеркальных антенн систем подвижной связи. Показано, что независимо от конструкции зеркала в заднем полупространстве на уровне фазового центра наблюдаются потоки мощчности, величина которых на два порядка превышает предельно допустимый уровень. Приводятся результаты визуализации распределения плотности потока энергии, показываются области их возможного использования.

Ключевые слова: плотность потока энергии, зеркальные антенны, визуализация структуры поля, круглая апертура, заднее полупространство, область дифракции.

Одна из причин роста систем телекоммуникации в мире, а также в России, обусловлена расширением спектра предоставляемых услуг, основной из которых

является широкополосный доступ к сети Интернет, включающий не только передачу данных, но и голоса (услуги Voice over Wi-Fi и VoLTE), изображения (Интернет-ТВ) и др. [1,
\end{abstract}




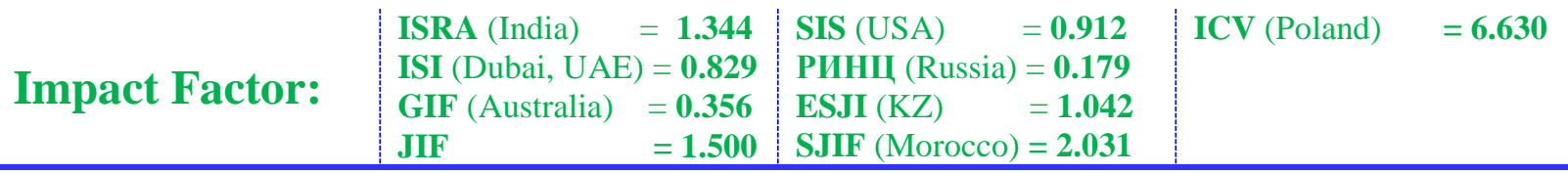

c.12]. Подтверждением этого являются статистические данные, приведенные в [2]. Так, в России в 2012 году доход отрасли составил 679,2 млрд. руб., причем $40 \%$ этой суммы приходилось на доходы от предоставления услуг мобильной связи. В стране наблюдается рост числа абонентских устройств подвижной связи (мобильных телефонов, смартфонов, планшетных компьютеров с выходом в
Интернет через сети сотовой связи) на 1000 человек населения. На Рис.1 вынесена гистограмма изменения данного показателя в период с января 2010 года по январь 2015 года по округам Российской Федерации и по стране в целом. Кроме того, растет число пользователей мобильного Интернета, подтверждающийся данными рис. 2.

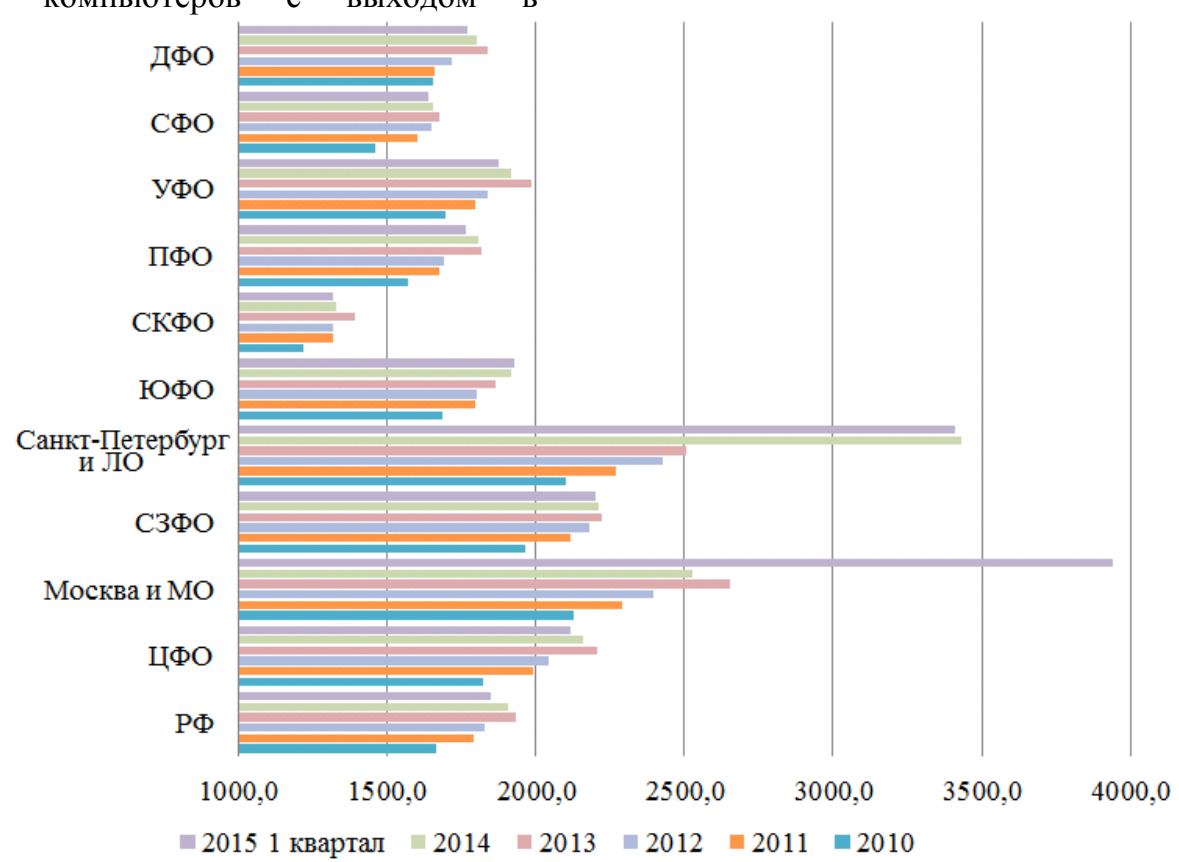

Рисунок 1 - Изменение числа абонентских устройств сотовой связи на 1000 человек населения в период 2010-2015 гг.

Проникновение, осень 2013,\% — Прирост, 2014

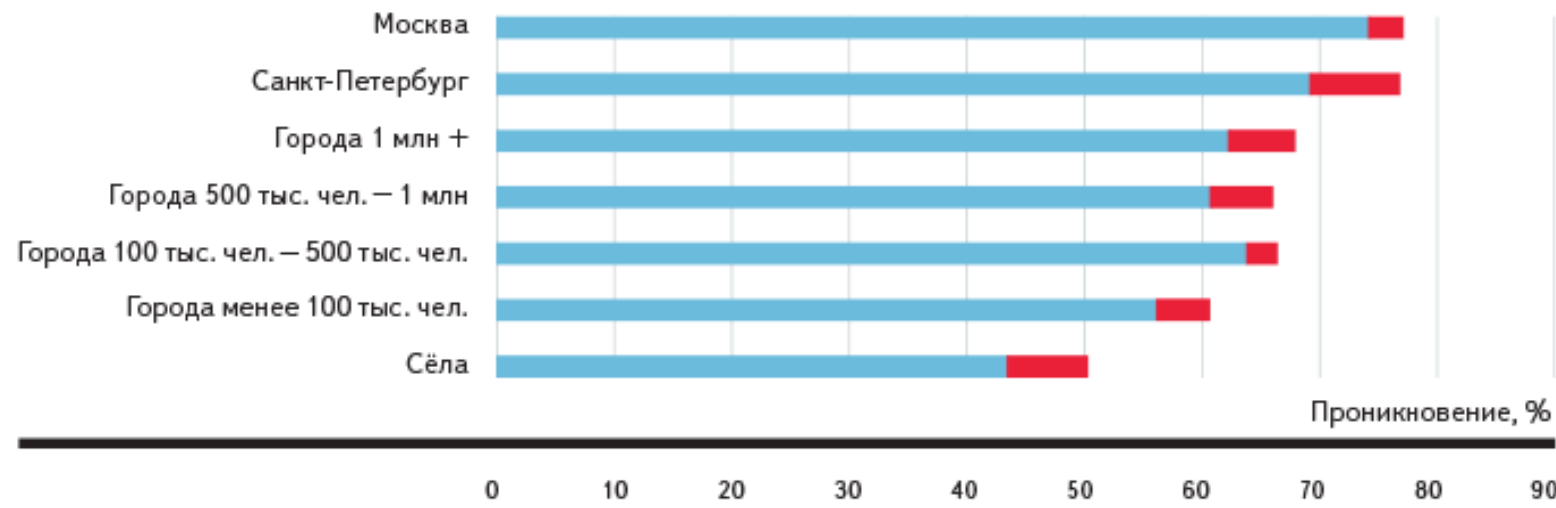

Рисунок 2 - Проникновение Интернет по типам населенных пунктов в 2014 году.

Анализ данных, приведенных на рис. 1, показывает, что в экономически развитых районах за последние пять лет рост устройств мобильной связи составил почти 100\%. При этом, как следует из анализа рис. 2 , основное их число пользователей мобильного Интернета приходится на крупные города с населением от
100 тыс. жителей. Расширение абонентской аудитории, а также расширение спектра предоставляемых услуг привело к росту числа базовых станций подвижной связи. Так, по данным [2] общее число базовых станций мобильной связи на декабрь 2013 года составило 82 211, в том числе старого стандарта 
$2 G$ - 29961 , действующего стандарта $3 G$ 30753 , нового стандарта $4 G-21497$. Средняя плотность размещения базовых станций в России составляет 11 единиц на 1 квадратный километр, а в районах повышенного проникновения данный показатель доходит до 90 единиц на 1 квадратный километр.

Негативным последствием роста числа систем подвижной связи является существенное усиление в крупных городах электромагнитного фона. Как показано в работе [3, с.4], профилактика электромагнитного загрязнения окружающей среды затруднены следующими факторами:

- невозможностью ограничения выброса загрязняющего фактора в окружающую среду невозможно, поскольку излучение - основное средство для переноса информации у передающих антенн, а также снижения электромагнитного поля до природного фона;

- долговременным воздействием в городских условиях электромагнитного поля (круглосуточно и даже на протяжении ряда лет) на большие контингенты людей, включая детей, стариков и больных;

- невозможностью статистического описания параметров излучений многих источников, распределённых в пространстве и имеющих различные режимы работы, в результате чего воздействие на население должно рассматриваться и как суммарный электромагнитный фон (интегральный показатель) и как воздействие сильных электромагнитных полей от отдельных источников.
Сказанное выше делает актуальным наблюдение за воздействующим фактором, а также прогнозирование обстановки по данному фактору, т.е. ведение электромагнитного мониторинга окружающей среды. Для населения крупных городов, где базовые станции систем подвижной связи размещаются на селитебной территории, особо актуальным становится социально ориентированный электромагнитный мониторинг, основной целью которого является своевременное и оперативное информирование населения об уровне электромагнитного загрязнения той или иной территории, с наглядной визуализацией полученных данных [4, с.40].

Излучающими в системах подвижной связи являются антенны базовых станций, антенны дуплексных каналов спутникового интернета, а также антенны с поддержкой $2 \times M I M O$ для создания мостов «точка-точка» при передаче данных в беспроводных сетях на расстояния от 9 до 200 км [5]. Типовыми конструкциями антенн при этом являются вибраторные, панельные, a также зеркальные антенны. Вопросы визуализации распределения излучаемой мощности антенн, установленных на крыше высотных зданий, рассматривались в работах авторов [6-9]. В связи с этим рассмотрим вопросы визуализации излучаемой мощности для зеркальных антенн с круглой апертурой. Электрические параметры типовых зеркальных антенн приведены в табл.1, составленной по данным [10].

Таблица 1

Электрические параметры типовых зеркальных антенн систем подвижной связи.

\begin{tabular}{||l|c|c|c|c||c||}
\hline Модель антенны & $\begin{array}{c}\text { Частотный } \\
\text { диапазон, } \\
\text { МГц }\end{array}$ & $\begin{array}{c}\text { Коэффициент } \\
\text { усиления } \\
\text { антенны, дБ }\end{array}$ & $\begin{array}{c}\text { Максимально } \\
\text { допустимая } \\
\text { входная мощность, } \\
\text { Вт }\end{array}$ & $\begin{array}{c}\text { Диаметр } \\
\text { зеркала, м }\end{array}$ & $\begin{array}{c}\text { Конструкция } \\
\text { зеркала }\end{array}$ \\
\hline RD-5G30-LW, Ubiquiti & $5,15-5,9$ & 30 & 100 & 0,65 & сплошное \\
\hline $\begin{array}{l}\text { MTAD-5G-3-D3 } \\
\text { (mANT30), Mikrotik }\end{array}$ & $4,7-5,9$ & 30 & 100 & 0,7 & сплошное \\
\hline ASPD-5.3-37 & $5,15-6,4$ & 37 & 50 & 1,44 & $\begin{array}{c}\text { проволочная } \\
\text { сетка }\end{array}$ \\
\hline \hline AF-5G34-S45, Ubiquiti & $5,15-6,4$ & 34 & 100 & 1,05 & сплошное \\
\hline RD-5G-34, Ubiquiti & $4,9-5,9$ & 34 & 100 & 0,972 & сплошное \\
\hline$R D-2 G-24$, Ubiquiti & $2,3-2,7$ & 24 & 50 & 0,65 & сплошное \\
\hline \hline$A F-2 G 24-S 45$, Ubiquiti & $2,3-2,7$ & 24 & 100 & 0,65 & сплошное \\
\hline
\end{tabular}




\begin{tabular}{|c|c|c|c|c|c|c|}
\hline Impact Factor: & $\begin{array}{l}\text { ISRA (India) } \\
\text { ISI (Dubai, UAF } \\
\text { GIF (Australia) } \\
\text { JIF }\end{array}$ & $\begin{array}{l}=1.344 \\
=0.829 \\
=0.356 \\
=1.500\end{array}$ & $\begin{array}{l}\text { SIS (USA) } \\
\text { PИНЦ (Russia) } \\
\text { ESJI (KZ) } \\
\text { SJIF (Morocco) }\end{array}$ & $\begin{array}{l}=0.912 \\
=0.179 \\
=1.042 \\
=2.031\end{array}$ & ICV (Poland) & $=6.630$ \\
\hline
\end{tabular}

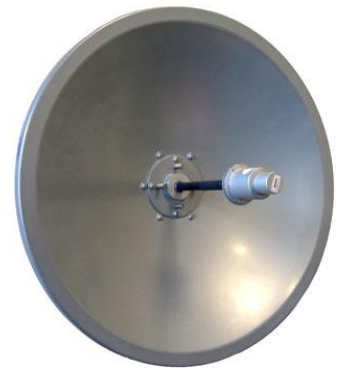

a)

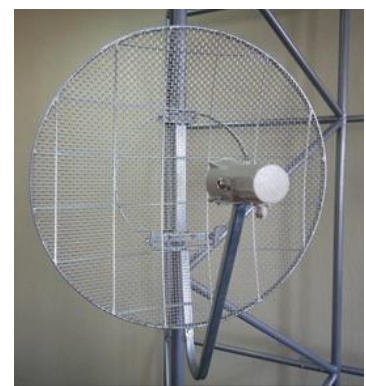

б)

Рисунок 3 - Конструкции зеркала антенны подвижной связи: a - сплошная цельнометаллическая; б - из проволочной сетки.

Анализ данных табл.1 показывает, что подводимая к антеннам мощность небольшая и колеблется от 50 до 100 Вт в зависимости от необходимости обеспечения дальности моста. Конструкция зеркала, как следует из рис.3, может быть выполнена двумя способами - в виде сплошного металлического зеркала и с помощью сетки из проволоки.

Для одного из типов антенн с отражающим экраном - панельных - в работе [6, с.14-15] было показано, что наличие экрана практически исключает излучение в заднее полупространство. В связи с этим вполне закономерным является вопрос оценки электромагнитной обстановки апертурной антенны в её заднем полупространстве. При этом, как и в работах [6-9], визуализацию распределения плотности потока энергии в рамках социально ориентированного электромагнитного мониторинга следует осуществлять в двух плоскостях: горизонтальной и вертикальной. При использовании

«предупредительного принципа», рекомендуемого Всемирной организацией здравоохранения [11, с.727; 13], необходимо рассматривать наихудшие с точки зрения влияния на электромагнитную экологию направления излучения антенны. Для зеркальной антенны, как следует из теории, приведенной, например, в [12], максимальное излучение антенны ориентировано по лучу, перпендикулярному апертуре и проходящему через фазовый центр антенны. Таким образом, вертикальная плоскость должна проходить через фазовый центр антенны и направление максимального излучения. В горизонтальной плоскости визуализацию результатов целесообразно осуществлять, как минимум на двух высотах: на высоте размещения фазового центра антенны, где излучение антенны максимально, и на высоте 2 м над поверхностью Земли, где осуществляется оценка безопасности электромагнитной обстановки [12].

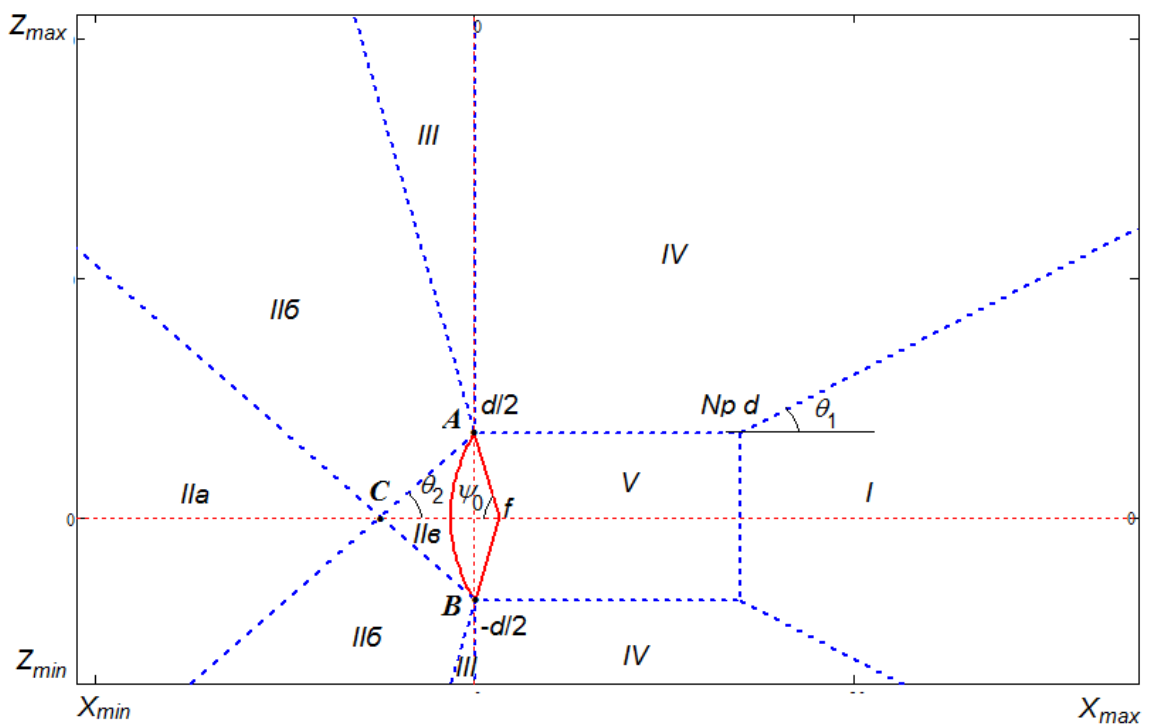

Рисунок 4 - Разделение пространства вокруг апертурной антенны на характерные зоны. 


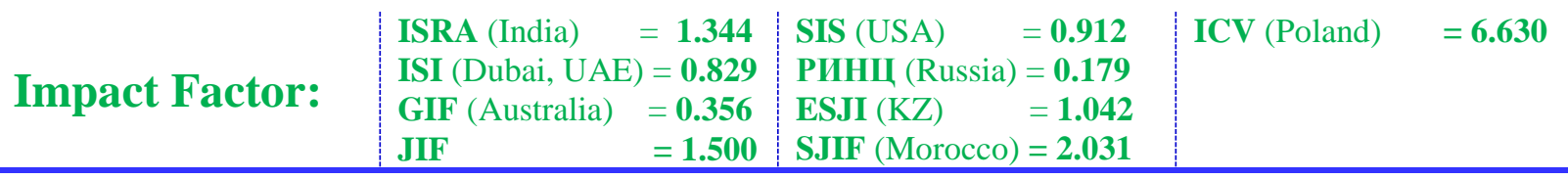

Для расчета распределения плотности потока энергии от зеркальной антенны с круглой апертурой используем «Методические указания...» [12]. В соответствии с ними в окружающем антенну пространстве выделяется, как показано на рис.4, пять зон, в каждой из которых для расчета используются решения известных электродинамических задач.

Поскольку конструкция антенны является симметричной, то разделение пространства на области справедливо в обоих сечениях вертикальном и горизонтальном.

Анализ данного рисунка показывает, что в области заднего полупространства антенны размещаются III и II области, причем вторая область в свою очередь разбивается еще на три подобласти. Данные области образуются после проведения двух касательных (верхней и нижней) в точках $A$ и $B$. Из подобласти IІа видна вся кромка антенны, из подобласти ІІб видна часть кромки, из подобласти IIв кромка не видна вообще.

Границы областей для длиннофокусного зеркала $\left(\psi_{0}<\pi / 2\right)$, используемого, как следует из рис. 3 , в качестве антенн систем подвижной связи, определяются соотношениями:

- области II:

$$
\begin{array}{ll}
x_{0}=0, & z_{0}=0.5 d, \\
x_{1}=X_{\text {min }}, & z_{1}=0.5 d+\left|X_{\min }\right| \operatorname{tg}\left(\psi_{0}\right),
\end{array}
$$

- между подобластями области II:

верхняя касательная к зеркалу:

$$
\begin{gathered}
x_{0}=-2 f-\left|Z_{\min }\right| 4 f / d, z_{0}=Z_{\text {min }}, \\
x_{1}=0, \\
z_{1}=0.5 d,
\end{gathered}
$$

нижняя касательная симметричная верхней относительно нулевого уровня,

- области III с областью IV

$$
x=0, \quad Z_{\min } \leq z \leq Z_{\max },
$$

$X_{\min }, Z_{\min }, Z_{\max }$ определяются из неравенств, задающих границы расчетной плоскости:

$$
\begin{gathered}
-N B \cdot d \leq x \leq N V \cdot d, \\
-H_{A} \leq z \leq N T \cdot d .
\end{gathered}
$$

Здесь $N V$ - горизонтальный размер в переднем полупространстве; NB горизонтальный размер в заднем полупространстве; $N T$ - вертикальный размер в верхнем полупространстве; $N p$ - размер прожекторной зоны. Остальные параметры антенны показаны на рис.4.

При таком разделении величина плотности потока энергии, создаваемого в областях заднего полупространства, будет определяться соотношениями [12]:

- в области III

$$
\Pi_{\Sigma}^{I I I}=\left\{\begin{array}{cc}
\Pi_{\text {обл }}+\Pi_{\partial и ф}^{I I \sigma}, & \psi_{0}<\pi / 2, \\
\Pi_{a}+\Pi_{\partial и \phi}^{I I a}, & \psi_{0} \geq \pi / 2 .
\end{array}\right.
$$

- в области II

$$
\begin{aligned}
\Pi_{\Sigma}^{I I} & =\Pi_{\partial и \phi}+ \\
& + \begin{cases}\Pi_{n p}, & \text { решетчатое зеркало, } \\
0, & \text { сплошное зеркало, }\end{cases}
\end{aligned}
$$

Здесь $\Pi_{a}$ - апертурная составляющая;

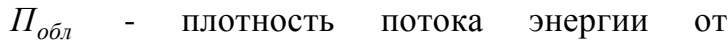
облучателя. Соотношения для их нахождения приведены в [12] и здесь не рассматриваются. $\Pi_{n p}$ - вклад энергии, прошедшей сквозь основное зеркало антенны, если оно имеет

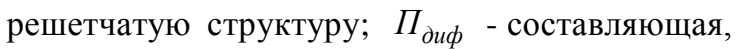
обусловленная токами, протекающими по кромке зеркала (дифракционная

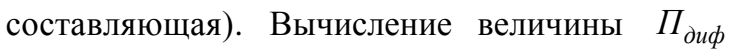
осуществляется по различным формулам. Так, в подобласти IIв величина $\Pi_{\partial и ф}=0$. Для

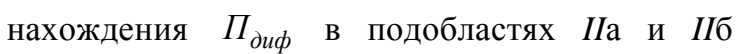
используются метод геометрической теории дифракции. В соответствии с ним, как показано в [12], напряженность поля параболической антенны в области тени $E_{T}$ определяется в основном напряженностью поля краевых дифракционных лучей, возникающих при падении волны, идущей от облучателя, на кромку зеркала:

$$
E=E_{0} \frac{\exp \left(-i \beta s^{\prime}\right)}{s^{\prime}} D_{1,2} \frac{\exp (-i \beta s)}{\sqrt{s}},
$$

где $E_{0}$ - амплитуда падающего поля; $s^{\prime}$ расстояние от точки источника до точки на ребре клина; $s$ - расстояние от точки на ребре клина до точки наблюдения; $D_{1,2}$ дифракционные коэффициенты для перпендикулярной и параллельной поляризаций.

При таком представлении поле в подобласти IIa определяются сферическими составляющими дифракционного поля, обусловленными всеми элементами ребра параболического зеркала. В подобласти ІІб значения сферических компонент дифракционного поля определяются только одной «светящейся» точкой ( $A$ или $B)$. Выражения для нахождения плотности потока энергии в данных подобластях являются достаточно громоздкими и подробно описаны в [12]. 


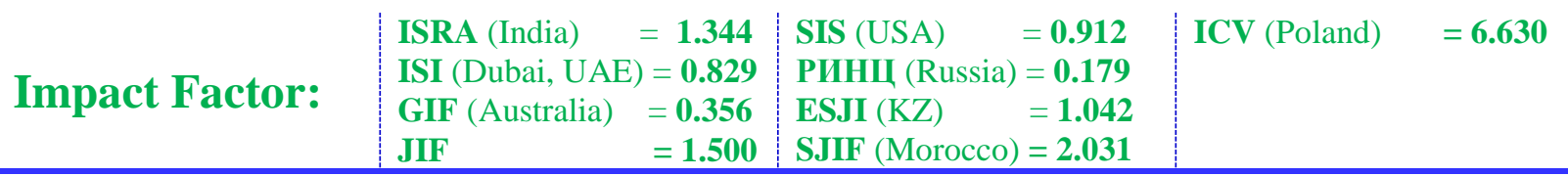

Для нахождения составляющей $\Pi_{n p}$, связанной с решетчатой структурой зеркала, используем соотношения, также приведенные в [12]. В соответствии с ними определяющим является коэффициент $T$ (по полю) прохождения волны через решетчатую структуру, зависящий от способа реализации зеркала. Для выполнения зеркала из проволочной сетки, как показано на рис. 3,б, данный коэффициент определяется формулой:

$$
\begin{aligned}
T= & \mid 1-\lambda \times \\
& \times\left(H_{0}^{(2)}(k \rho)+2 \sum_{n=1}^{N v} H_{0}^{(2)}(n k d s)\right)^{-1} \mid,
\end{aligned}
$$

где $N v$ - верхняя граница ряда разложения по функциям Ганкеля; $H_{0}^{(2)}(k \rho)$ - функция Ганкеля 2-го рода нулевого порядка, описывающая дифракцию на круглом проводе; $H_{0}^{(2)}(n k d s)$ дифракционная составляющая, позволяющая учесть множитель решетки; $2 \rho$ - диаметр провода $[\mathrm{M}] ; d s$ - шаг размещения проводов в сетке $[\mathrm{M}] ; \quad k=2 \pi c / f \quad$ - волновое число свободного пространства; $c=3 \cdot 10^{8} \quad$ [м/с] скорость света; $f$ - рабочая частота излучателя [Гц].

С использованием приведенных выше соотношений в пакете MATLAB были разработаны два сценария, позволяющие визуализировать распределение плотности потока энергии в заднем полупространстве зеркальной антенны с круглой апертурой. Корректность получаемых с использованием программ результатов была проверена для ряда частных случаев из [12].

Визуализацию распределения плотности потока энергии в заднем полупространстве проведем для антенны $R D-2 G-24$, функционирующей в диапазоне 2,4 ГГц. Угловой полураскрыв зеркала полагался равным $\psi_{0}=70^{\circ}$. На рис. 5 и 6 приведены результаты визуализации распределения плотности потока энергии в горизонтальной плоскости, проходящей через фазовый центр антенны для сплошной и сетчатой конструкций зеркала. На данных рисунках черным показан контур зеркала, штриховые линии разделяют область заднего полупространства.

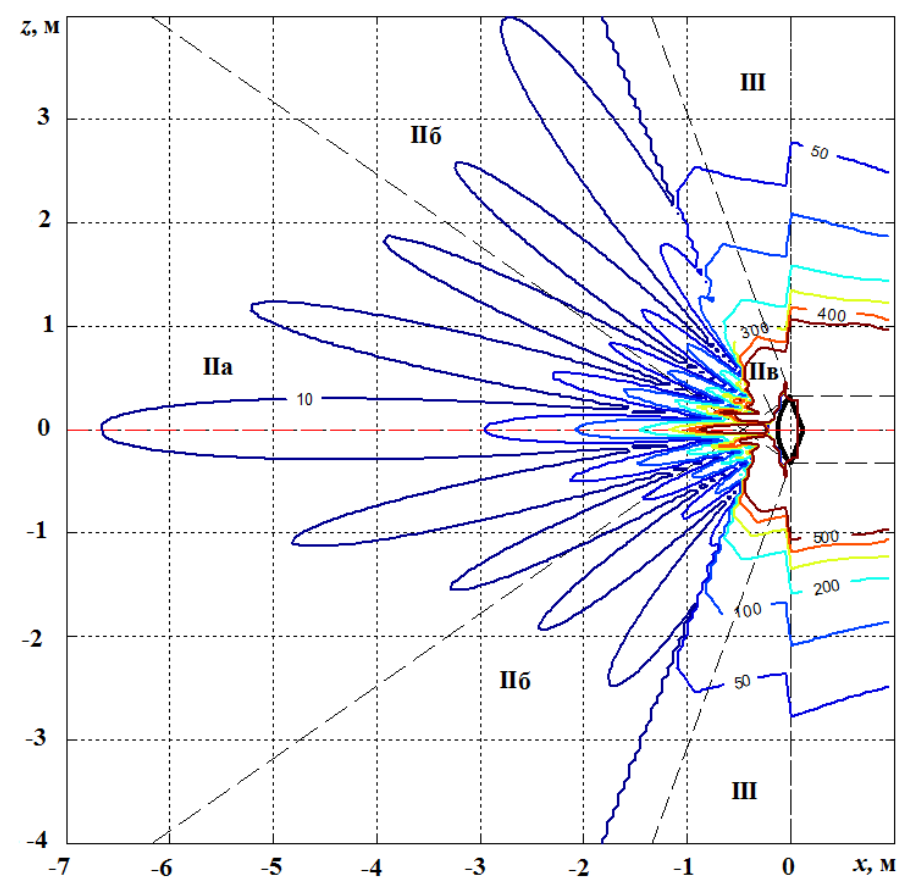

Рисунок 5 - Распределение плотности потока энергии в заднем полупространстве при сплошной конструкции длиннофокусного зеркала. 


\begin{tabular}{|c|c|c|c|c|c|c|}
\hline Impact Factor: & $\begin{array}{l}\text { ISRA (India) } \\
\text { ISI (Dubai, UAE } \\
\text { GIF (Australia) } \\
\text { JIF }\end{array}$ & $\begin{array}{l}=1.344 \\
=0.829 \\
=0.356 \\
=1.500\end{array}$ & $\begin{array}{l}\text { SIS (USA) } \\
\text { PИНЦ (Russia) } \\
\text { ESJI (KZ) } \\
\text { SJIF (Morocco) }\end{array}$ & $\begin{array}{l}=0.912 \\
=0.179 \\
=1.042 \\
=2.031\end{array}$ & ICV (Poland) & $=6.630$ \\
\hline
\end{tabular}

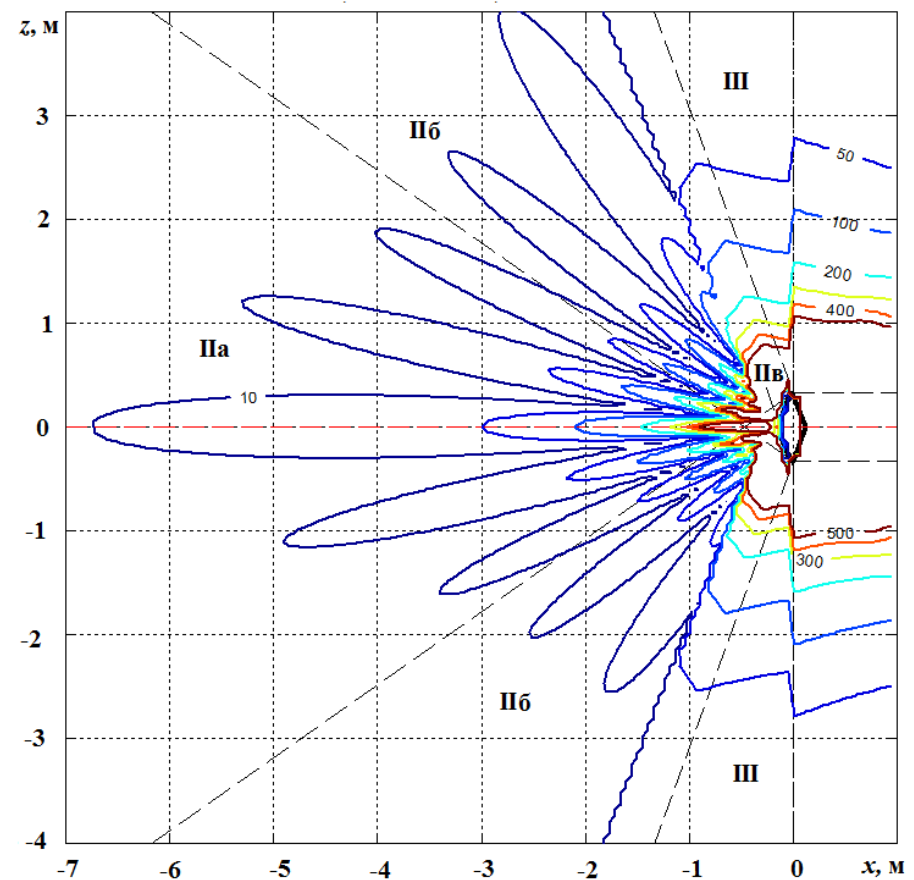

Рисунок 6 - Распределение плотности потока энергии в заднем полупространстве при сетчатой конструкции длиннофокусного зеркала.

Анализ полученных результатов показывает, что независимо от конструкции зеркала в заднем полупространстве в непосредственной близости от антенны имеют области, в которых плотности потока энергии на несколько порядков превышает предельно допустимый уровень в 10 мкВт/см². Максимальный размер санитарно-защитной зоны составляет порядка 7 м в направлении, противоположном направлению максимального излучения. Выполнение зеркала с использованием сетчатой конструкции формирует непосредственно за зеркалом (в подобласти ІІв) электромагнитное поле, плотность потока энергии в котором составляет порядка 300 мкВт/см². Максимальные размеры данного участка подобласти не превышают
0,5 м. В остальных подобластях распределение плотности потока энергии практически не зависит от исполнения зеркала и представляет собой интерференцию двух волн, затекающих с краев зеркала в область заднего полупространства. В результате чего наблюдаются интерференционные лепестки.

Для выявления влияния формы зеркала на распределение плотности потока энергии на рис. 7 и 8 приведены результаты визуализации при тех же параметрах, за исключением значения углового раскрыва зеркала. Рассмотрим теперь случай выполнения зеркала в виде короткофокусного параболоида вращения с $\psi_{0}=120^{\circ}$. 


\begin{tabular}{|c|c|c|c|c|c|}
\hline Impact Factor: & $\begin{array}{l}\text { ISRA (India) } \\
\text { ISI (Dubai, UAF } \\
\text { GIF (Australia) } \\
\text { JIF }\end{array}$ & $\begin{array}{l}=1.344 \\
=0.829 \\
=0.356 \\
=1.500\end{array}$ & $\begin{array}{ll}\text { SIS (USA) } & =\mathbf{0 . 9 1 2} \\
\text { PИНЦ (Russia) } & =\mathbf{0 . 1 7 9} \\
\text { ESJI (KZ) } & =\mathbf{1 . 0 4 2} \\
\text { SJIF }(\text { Morocco) } & =\mathbf{2 . 0 3 1}\end{array}$ & ICV (Poland) & $=6.630$ \\
\hline
\end{tabular}

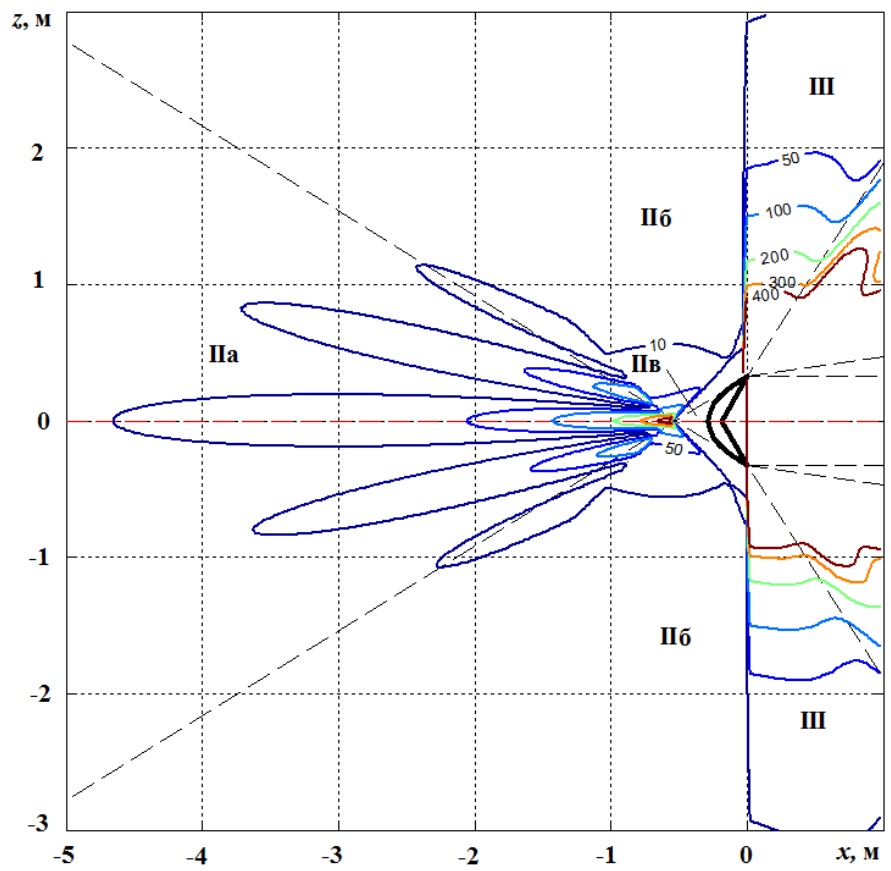

Рисунок 7 - Распределение плотности потока энергии в заднем полупространстве при сплошной конструкции короткофокусного зеркала.

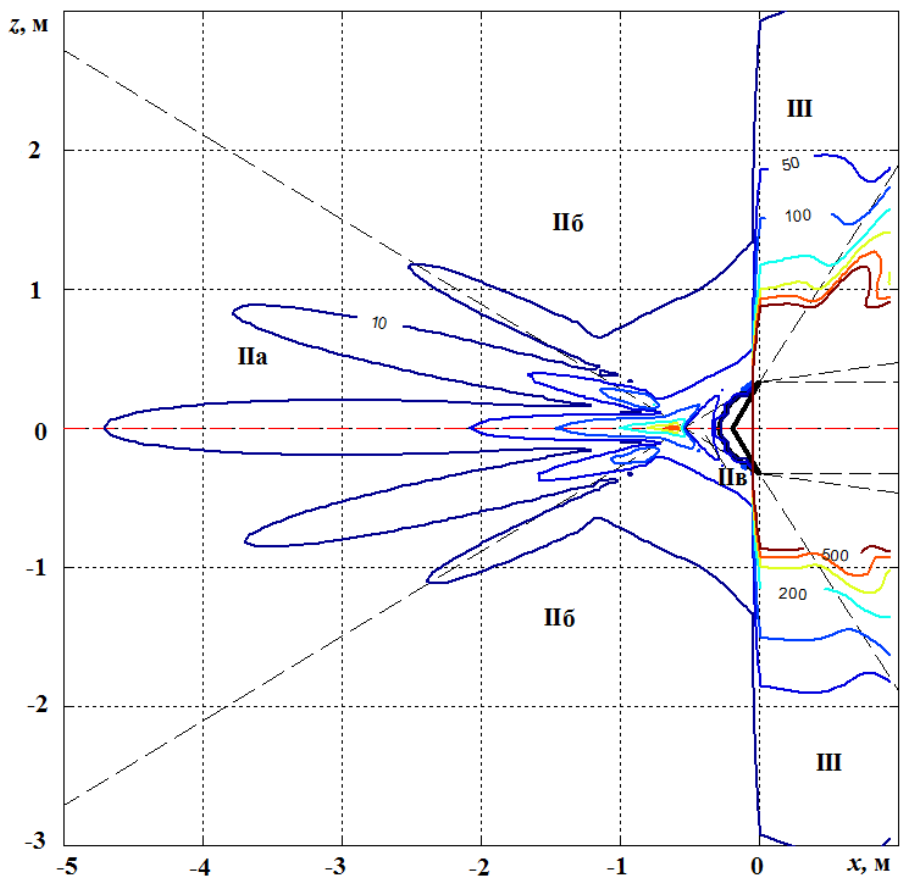

Рисунок 8 - Распределение плотности потока энергии в заднем полупространстве при сетчатой конструкции короткофокусного зеркала.

Анализ результатов, приведенных на рис.7 и 8 , а также их сравнение с данными рис. 5 и 6 , показывает, что при большей глубине зеркала и размещении облучателя внутри апертуры амплитуда дифракционных полей, возбуждаемых на кромках зеркала, а. следовательно, и волн, затекающих в заднее полупространство, существенно ниже, чем для длиннофокусной антенн. В результате максимальный размер санитарно-защитной зоны в заднем полупространстве меньше и составляет не 7 м, а всего 4,5 м. Меньше становятся и амплитуды интерференционных лепестков поля. Выполнение зеркала в виде сетки также приводит к просачиванию поля в область задней полусферы в подобласть ІІв. 


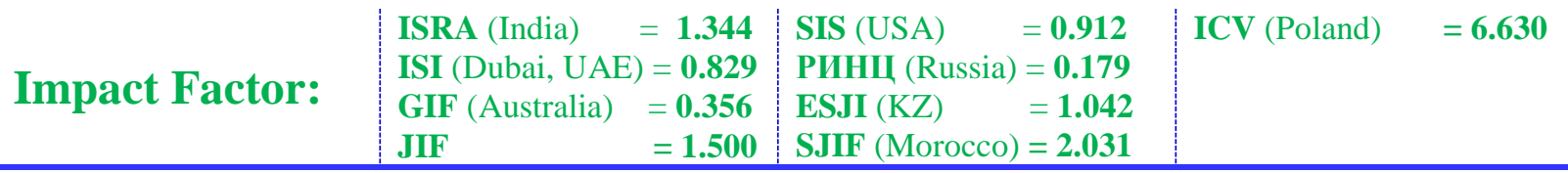

Однако уровни плотности потока энергии в данной подобласти не превышают предельно допустимые значения. В подобласти IIa, в которой в плотность потока энергии вносят вклады две противоположно направленные волны от всех точек кромок, как и для длиннофокусного зеркала наблюдается превышение уровня поля на два порядка по сравнению с величиной предельно допустимого значения.

Таким образом, получаемые с использованием разработанных авторами программ результаты обеспечивают визуализацию электромагнитной обстановки вблизи места размещения зеркальной антенны с круглой апертурой, используемой в системах подвижной связи. Получаемая визуализация обеспечивает не только понятные для неподготовленного пользователя результаты в рамках социально ориентированного электромагнитного мониторинга, но и позволяет специалистам давать их физическую трактовку, a, следовательно, использовать получаемую информацию при проектировании антенн, удовлетворяющих требованиям электромагнитной экологии.

\section{References:}

1. (2015) Telekommunikatsii 2015 - prognoz, Tekhnologii i sredstva svyazi, №1, pp.12-13.

2. Effektivnost' ekonomiki Rossii, Available: http://www.gks.ru/wps/wcm/connect/rosstat_m ain/rosstat/ru/statistics/efficiency/\# (Accessed: 20.08.2015)

3. Dovgusha VV, Tikhonov MN, Dovgusha LV (2009) Vliyanie estestvennykh i tekhnogennykh elektromagnitnykh poley na bezopasnost' zhiznedeyatel'nosti, Ekologiya cheloveka, №12, pp.3-9.

4. Maslov MY, Spodobaev MY, Spodobaev YM (2014) Sovremennye problemy elektromagnitnoy ekologii, Elektrosvyaz', №10, pp.39-42.

5. (2015) Internet-magazin O-t-s.ru, Available: http://www.o-t-s.ru/all_equipments_3.html (Accessed: 22.08.2015).

6. Zvezdina MY, et al. (2015) Electromagnetic situation visual representation near mobile base station antenna, Theoretical \& Applied Science, № $\quad 3(23), \quad$ pp.10-17. doi: http://dx.doi.org/10.15863/TAS.2015.03.23.3

7. Zvezdina MY, et al. (2015) Visual representation of energy flux density for mobile system aerial in MathCad, Modeling of Artificial Intelligence, Vol.5, №1. p.42-48. Available:

http://ejournal11.com/journals_n/1428676707.p df doi: 10.13187/mai.2015.5.42 (Accessed: 20.08.2015)

8. Zvezdina MY, et al. (2015) Modelirovanie elektromagnitnoy obstanovki vblizi antenny sistemy podvizhnoy svyazi na kryshe zhilogo doma, Trudy Severo-Kavkazskogo tekhnicheskogo universiteta svyazi i informatiki, Part 2, Rostov n/D: PTs "Universitet", 2015. pp.185-188.

9. Zvezdina MY, et al. (2015) Programmnyy kompleks dlya vizualizatsii elektromagnitnoy obstanovki vblizi antenny sotovoy svyazi. Sbornik nauchn. trudov po materialam Mezhdunarodnoy nauchno-prakticheskoy konferentsii «Teoreticheskie i prakticheskie voprosy nauki i obrazovaniya». Tambov, January, 31, 2015г. Part 10. Tambov: OOO "Konsaltingovaya kompaniya Yukom ", 2015. 164 p.; pp.70-74, (Date of access: 08.08.2015).

10. (2015) Sayt LanMarket "Ves' mir setevogo oborudovaniya v odnom magazine", Available: http://lanmarket.ua (Accessed: 20.08.2015)

11. Grigor'ev YG, Grigor'ev KA (2005) Elektromagnitnye polya bazovykh stantsiy podvizhnoy radiosvyazi i ekologiya. Otsenka opasnosti elektromagnitnykh poley bazovykh stantsiy dlya naseleniya i bioekosistem, Radiatsionnaya biologiya. Radioekologiya, Vol.45, № 6, pp.726-731.

12. (2003) MUK 4.3-1167-02. Opredelenie plotnosti potoka energii elektromagnitnogo polya $\mathrm{v}$ mestakh razmeshcheniya radiosredstv, rabotayushchikh v diapazonakh chastot 300 MGts - 300 GGts. Utv. Predsedatelem Gos. sanitarno-epidemiologicheskogo nadzora RF 7.10.2002, Available: http://rfcmd.ru/sphider/docs/project/MUK\%204 .3.1167-02.htm (Accessed: 07.08.2015). 